Bartosz Kania $^{\star}$, Tomasz Dziwiński ${ }^{\star \star}$, Waldemar Bauer ${ }^{\star \star}$, Marta Zagórowska ${ }^{\star \star}$, Jerzy Baranowski ${ }^{\star \star}$

\title{
A Comparison between Integer Order and Non-integer Order Controllers Applied to a Water Levelling System
}

\section{Introduction}

Recently, one can see an increasing interest in analysis and implementing non-integer order controllers $[1,2,3,4]$. Most of them relate to controlling complex systems and the efficiency of the non-integer order controllers is often better for such objects. We wanted to investigate if the non-integer order $\mathrm{PI}^{\lambda}$ controller has better performance than a classical PI controller for a nonlinear simple integrating system.

The considered system consists of two tanks (one of them is a buffer tank), a valve, a level sensor, a pipe system and a pump enforcing a movement of water [5]. The pump is driven by a DC motor, which is controlled by a PWM signal. The valve is positioned manually. The level sensor ensures a feedback loop. This system is presented in Figure 1.

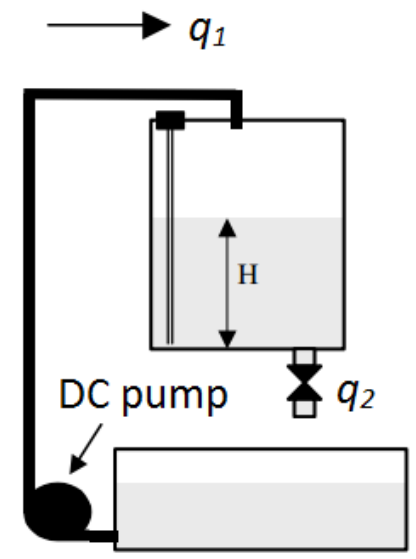

Fig. 1. Simplified controlled system

\footnotetext{
* Lublin University of Technology, Department of Automatics and Metrology, Lublin, Poland, email: b.kania@pollub.pl

${ }^{\star \star}$ AGH University of Science and Technology, Department of Automatics and Biomedical Engineering, Krakow, Poland, e-mail: tdz@agh.edu.pl, bauer@agh.edu.pl,zagor@agh.edu.pl,jb@agh.edu.pl
} 
The considered system has the following parameters:

$a=0.31 m-$ width

$w=0.5 m-$ depth

$H_{\text {max }}=0.4 m-$ maximal height

This system is nonlinear because of the nonlinear function of the valve flow in dependence of water level in tank.

\section{Modelling}

A diagram of a water level automatic control system is illustrated in Figure 2. It is a classic approach to automatic control system. A reference value $r(t)$ is added to an output signal and it goes to the controller as an error signal $e(t)$. The controlling signal $u(t)$ appears at the controller output and controls the pump directly. The pump is a part of controlled system. The loop feedback is realized by the level sensor.

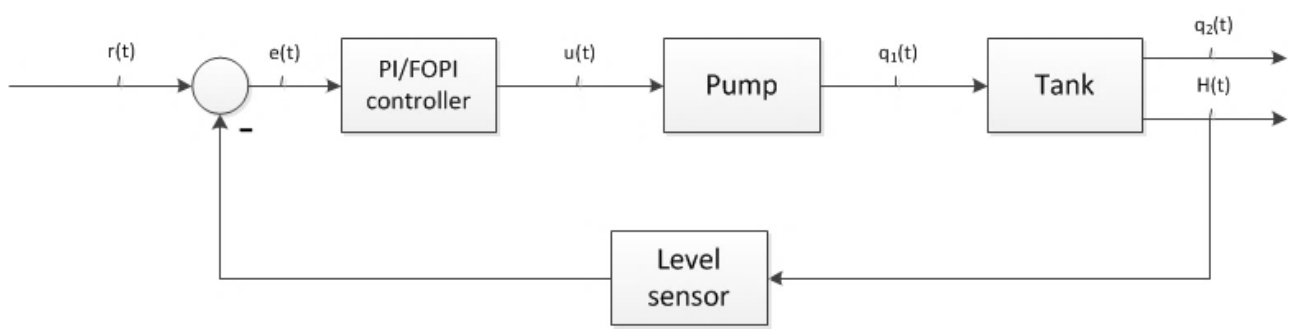

Fig. 2. Diagram of an automatic control system

The control input and output of the system is the water level measured in the tank.

The parameters of considered model have been identified by the experiments on the above-mentioned hydro-mechanical system.

The modelling process started with obtaining necessary characteristics. This means:

- the water level $H$ in function of the output sensor voltage,

- the pump efficiency $q_{1}$ in function of the controlling signal $u$,

- the valve flow $q_{2}$ in function of the water level in tank $H$.

First of necessary functions is obtained in the following way: the tank was filled up to a known level and then the value of the output voltage was read (one hundred last samples) and an average value was calculated. This was repeated for a few known water levels. In a result the function of the water level in the tank in dependence of the output sensor voltage was obtained. It is presented in Figure 3. 


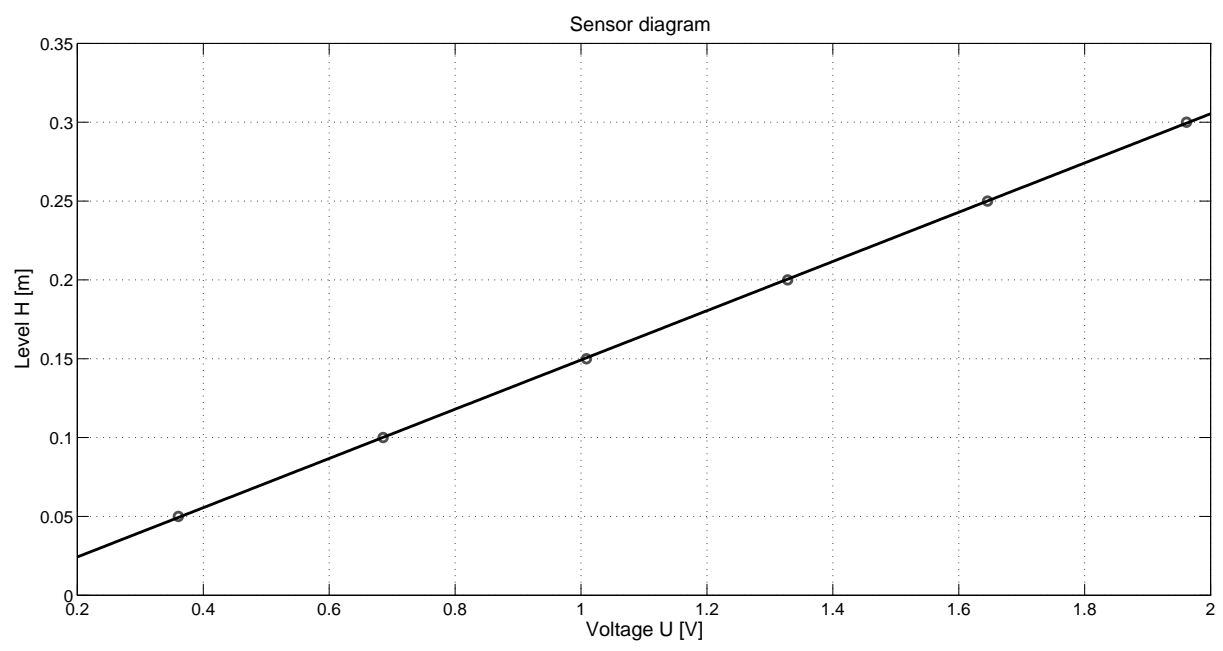

Fig. 3. Diagram of the level's sensor

The approximation of the above characteristic gives a following linear function:

$H(U)=0.15616 U+0.0069352$

The next important relationship is the pump efficiency in dependence of the controlling signal. The tank was emptied and the valve was closed. Then, the tank was filled up by given time $(T=20 \mathrm{~s})$ by the pump controlled with constant PWM signal whereupon the water level was read. This was repeated a few times for different, but constant PWM signals. As a result of those attempts we got set of water level values $H$. Volume of water $V(H)$ for each of attempts is given by:

$$
V(H)=a w H
$$

Finally, the pump efficiency $q_{1}$ is calculated by the following expression:

$$
q 1(H)=V(H) / T
$$

where $T=20 \mathrm{~s}$.

This function is illustrated in Figure 4 and can be approximated with polynomial function:

$$
q_{1}(H)=10-4 \cdot\left(3.01 \cdot H^{3}-6.52 \cdot H^{2}+5.83 \cdot H-0.701\right)
$$




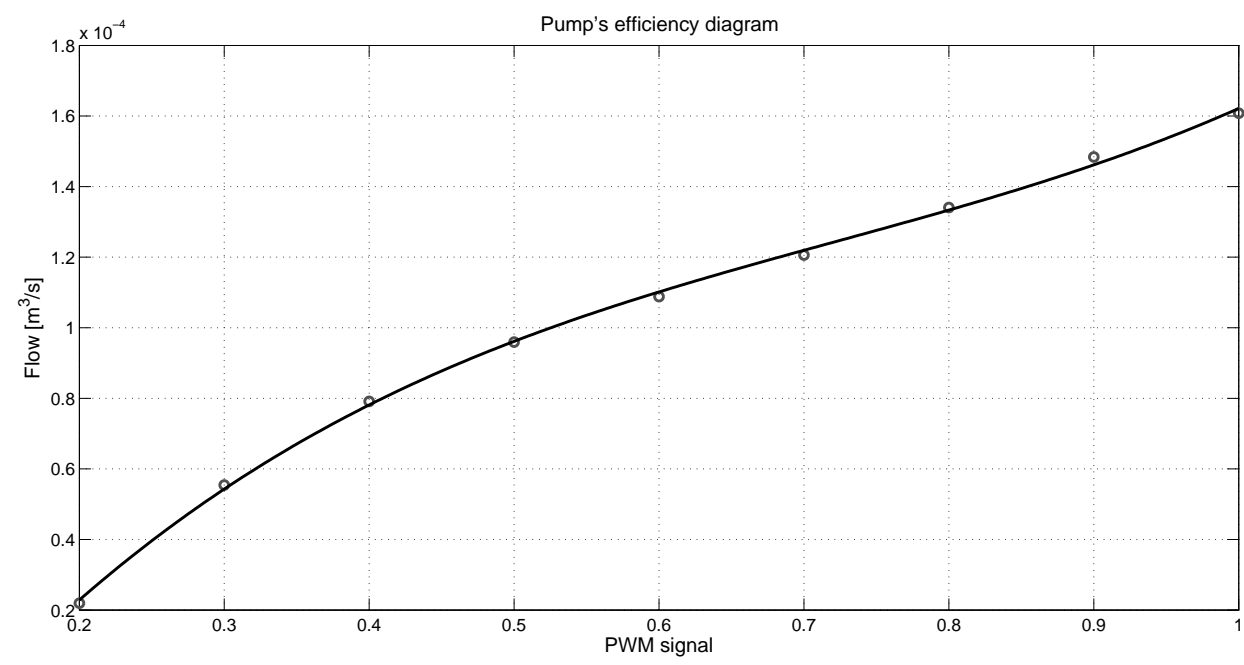

Fig. 4. The pump's efficiency diagram

The last necessary function is the valve flow in dependence of the water level. The valve was partly opened and blocked to obtain constant properties of the system and pump was controlled by a constant PWM signal for a long time (min. 5 minutes). The system was brought to a steady state. In this case, pump efficiency equals the valve flow. The function of pump efficiency is known (Fig. 4), so executing the above-mentioned attempts for different PWM signals gives a possibility to get the valve flow in the dependence of the water level, see Figure 5, pointed data.

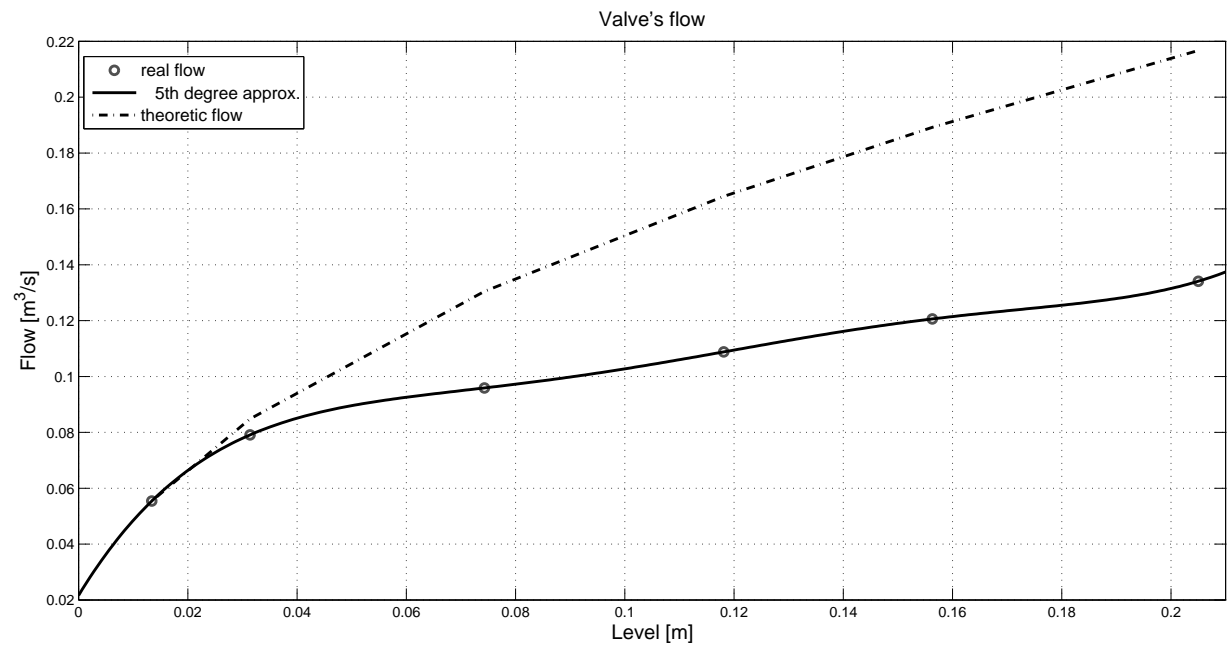

Fig. 5. The valve's diagram: approximation of real flow - continuous line; theoretic flow - dashdot line 
One can expect:

$$
Q_{2}=c \sqrt{\frac{p_{w}}{b}+H g-\frac{p_{z}}{b}}
$$

where:

$$
\begin{aligned}
& p_{w} \text { - a pressure affecting the water's surface, } \\
& p_{z} \text { - an external pressure, } \\
& b \text { - a fluid specific weight, } \\
& H \text { - a fluid column height, } \\
& g \text { - the gravity force, } \\
& c \text { - a constant. }
\end{aligned}
$$

Let us consider $p_{w}=p_{z}$, therefore, one can write:

$$
q_{2}(H)=c_{2} \cdot \sqrt{H}
$$

However, the obtained characteristic does not fit to a theoretic diagram, see Figure 5, so one can conclude that the valve's flow isn't laminar. The fifth order polynomial approximation seems to be a better representation:

$$
q_{2}(H)=4093 \cdot H^{5}-2479 \cdot H^{4}+564,9 \cdot H^{3}-59.99 \cdot H^{2}+3.231 \cdot H+0.0216
$$

The differential equation of the considered system is:

$$
\frac{d H}{d t}=\frac{1}{B} \cdot\left(q_{1}-q_{2}(H)\right)
$$

where:

$$
\begin{gathered}
B=a \cdot w-\text { the water's surface, } \\
q_{1}-\text { the pump efficiency, } \\
q_{2}(H)-\text { the valve flow. }
\end{gathered}
$$

The model of the controlled object and the controller is presented in Figure 6. There is a saturation added because of the limited volume of the tank.

Both the real system and the numerical model are nonlinear because of the nonlinearity in two of tree obtained system parts' functions. Controllers have been designed on the grounds of a reference point which has been chosen $\left(H_{0}=0.1 \mathrm{~m}\right)$. 


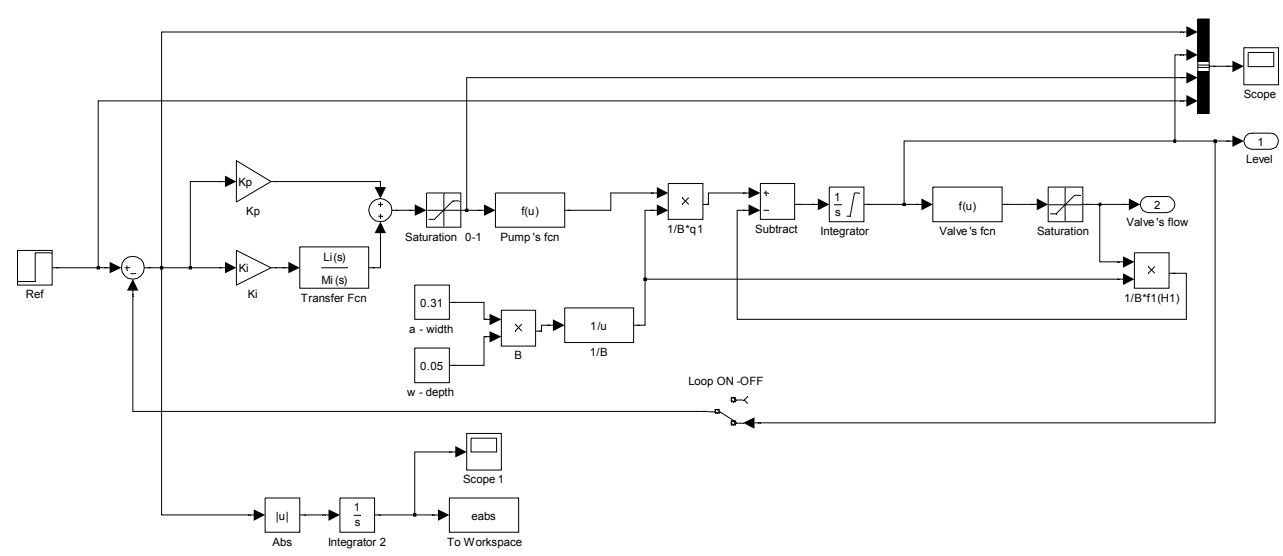

Fig. 6. Controlling system numerical model

\section{Controlling methods}

The non-integer order $\mathrm{PI}^{\lambda}$ controller is a simplified $\mathrm{PI}^{\lambda} \mathrm{D}^{\mu}$ and is described by an equation (7):

$$
G_{F O P I}(s)=K_{p}+K_{i} \frac{1}{s^{\lambda}}
$$

where $K_{p}, K_{i}$ and $\lambda$ denote proportional gain, integral gain and integral order respectively.

The comparison has been conducted using the following performances:

- steady state error cancellation,

- integral absolute error minimization.

In order to compare the controlling quality of two types of controllers we decided to choose the optimisation method, which gave good results in previous research, it is the simulated annealing [1,2] method. This minimization method is able to find a global minimum of an objective function. In this case, the integrated absolute error has been chosen as the objective function (8).

This minimization method is able to find a global minimum of an objective function. In this case, the integrated absolute error has been chosen as the objective function (8).

$$
I A E=\int_{0}^{t}|e(t)| d t
$$

Our purpose is to find the minimum of the objective function for both of the controllers using numerical models of the control system and a real-time simulation results. 
In Figure 6 the considered system's model with the non-integer order $\mathrm{PI}^{\lambda}$ controller is presented. If we set $\lambda=1$, this controller becomes the traditional PI controller. An optimisation process is made by the simulated annealing method using the parameters from Table 1.

Table 1

The parameters of the optimisation

\begin{tabular}{|l|c|c|l|}
\hline Parameter & PI & FOPI & Description \\
\hline$T_{s}$ & $100 \mathrm{~s}$ & $100 \mathrm{~s}$ & Simulation time \\
\hline$K p_{\min }$ & 1 & 1 & Minimum proportional coefficient \\
\hline$K i_{\min }$ & 0 & 0 & Minimum integral coefficient \\
\hline$\lambda_{\min }$ & 1 & 0.1 & Minimum integrator's order \\
\hline$K p_{\max }$ & 200 & 200 & Maximum proportional coefficient \\
\hline$K i_{\max }$ & 200 & 200 & Maximum integral coefficient \\
\hline$\lambda_{\max }$ & 1 & 0.9 & Maximum integrator's order \\
\hline$R e f$ & $0.1 \mathrm{~m}$ & $0.1 \mathrm{~m}$ & Reference value $\left(H_{0}\right)$ \\
\hline$U_{\min }$ & 0 & 0 & Minimum controlling signal \\
\hline$U_{\max }$ & 1 & 1 & Maximum controlling signal \\
\hline$H_{\max }$ & $0.35 \mathrm{~m}$ & $0.35 \mathrm{~m}$ & Maximum water's level \\
\hline$N$ & - & 5 & Oustaloup's approximation order \\
\hline$\omega_{\min }$ & - & $10^{-6}$ & Minimum approximation frequency \\
\hline$\omega_{\max }$ & - & $10^{2}$ & Maximum approximation frequency \\
\hline
\end{tabular}

There are two variables of the optimisation method in the PI controller case, those are a proportional coefficient $K_{p}$ and an integral coefficient $K_{i}$. In the case of the non-integer order $\mathrm{PI}^{\lambda}$ controller there are three variables, two first same as before and $\lambda$ as the third one. The non-integer order integrator is numerically approached using an Oustaloup approximation $[6,7]$. The Oustaloup continuous integer approximation is given by the equation:

$$
s^{\lambda} \approx K \prod_{i=1}^{N} \frac{s+\omega_{i}^{\prime}}{s+\omega_{i}}, \alpha>0
$$

where poles, zeros and gain can be evaluated respectively as:

$$
\begin{aligned}
& \omega_{i}^{\prime}=\omega_{\min } \omega_{u}^{(2 i-1-\alpha) / N} \\
& \omega_{i}=\omega_{\min } \omega_{u}^{(2 i-1+\alpha) / N}
\end{aligned}
$$




$$
\begin{aligned}
& \omega_{u}=\sqrt{\frac{\omega_{\max }}{\omega_{\min }}} \\
& K=\omega_{h}^{\alpha}
\end{aligned}
$$

To find optimal parameters of the controller, the simulated annealing optimization method has been chosen. Simulated Annealing is a minimization technique for solving an unconstrained and a bound-constrained optimization problem, which gives a good results in finding a local minimum. At each iteration of the Simulated Annealing algorithm, a new point is randomly generated. The distance of the new point from the current point is based on a probability distribution (temperature function) with a scale proportional to the temperature. The algorithm accepts all new points that lower the objective function value, but also, with a certain probability, points that raise the objective function value. By accepting points that raise the quality function, the algorithm avoids being trapped in local minima in early iterations and is able to explore globally for better solutions.

The above-mentioned Oustaloup approximation gives satisfactory results within the frequency range $[1,2]$. Consequently of carried optimisation process the following results are obtained, see Table 2.

Table 2

The results of the optimisation

\begin{tabular}{|c|c|c|}
\hline Coefficient & PI & FOPI \\
\hline$K_{p}$ & 189.86 & 157.143 \\
\hline$K_{i}$ & 0.5308 & 0.859 \\
\hline$\lambda$ & - & 0.8973 \\
\hline
\end{tabular}

\section{Experiments}

There have been conducted experiments to compare controller performance. A laboratory workplace that we use consists of a PC with the Matlab/Simulink environment, a RTDAC process board and a device driver. We added a FIR filter to the real-time controlling system to clear high frequency disturbances from the level sensor. It calculates a moving average with 40 last samples. Controller performance for the hydro-mechanical system have been investigated based on the following experiment. Firstly, we attempted to run the controlling process at simulation conditions what is illustrated in Figure 7. There one can see a short delay at the starting point resulting from a short water transportation delay in the pipe from the pump to the tank inlet. A filling process has been running correctly and stable till the water level reached the reference value. Then, the level in the tank has stabilized, but some fluctuation not eliminated in both cases. It is probably effect of water waves in the tank. 
Responses of the PI controller and the FOPI controller are very similar. The same can be seen in the error diagram (Fig. 8 and Fig. 9).

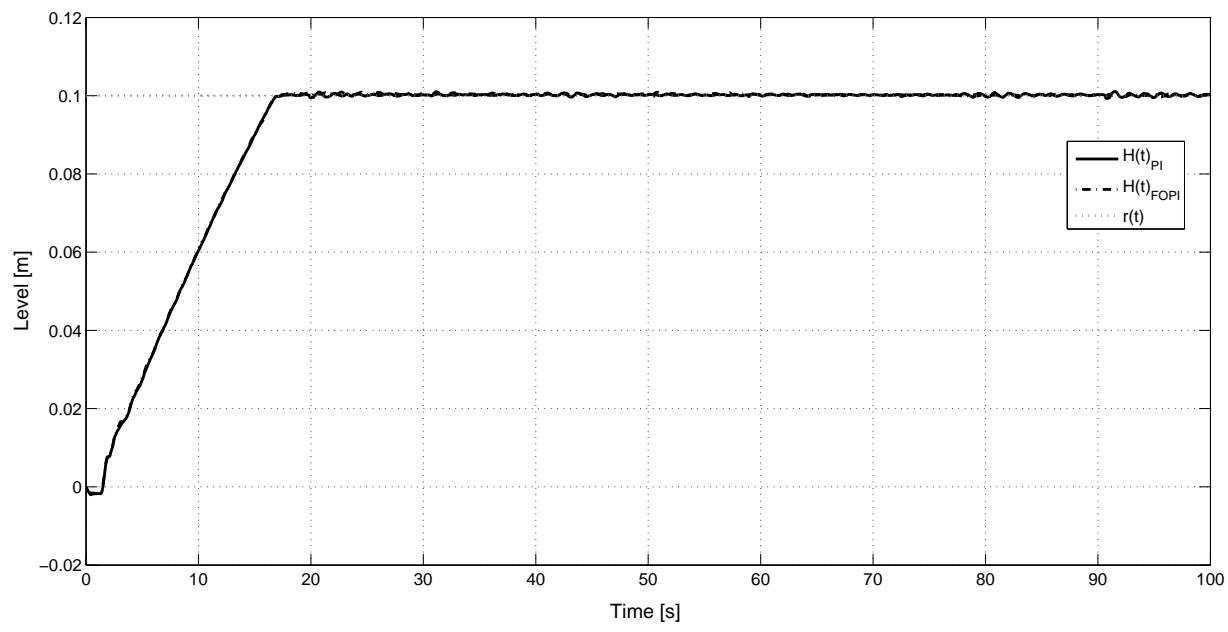

Fig. 7. Step responses for both of the controllers

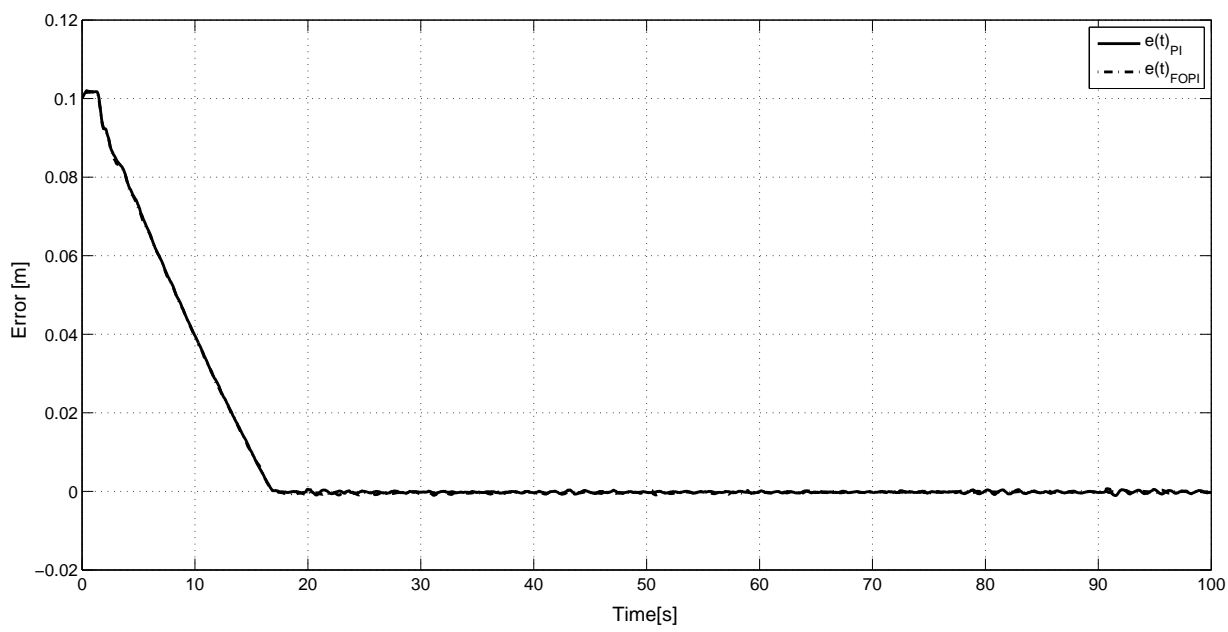

Fig. 8. The error signal for step response 


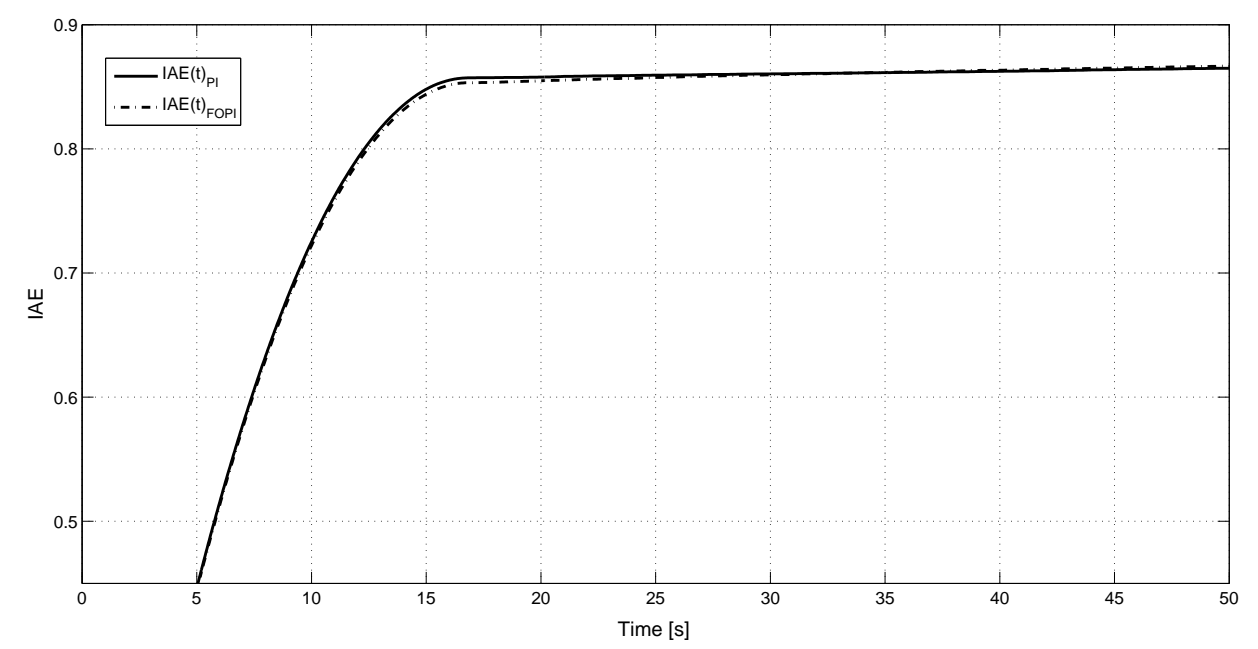

Fig. 9. The Integrated Absolute Error for step response

Secondly, the system response for different values of the reference signal has been investigated. One can find transient step responses for $0.1 \mathrm{~m}, 0.05 \mathrm{~m}, 0.15 \mathrm{~m}, 0.1 \mathrm{~m}, 0.2 \mathrm{~m}$ respectively in Figure 10. The reference value changed every 30 seconds. There is a little overshoot during the decreasing the water level and one can see that if the level is higher, the steady state error is larger. Both PI and FOPI controllers offer an almost identical time response.

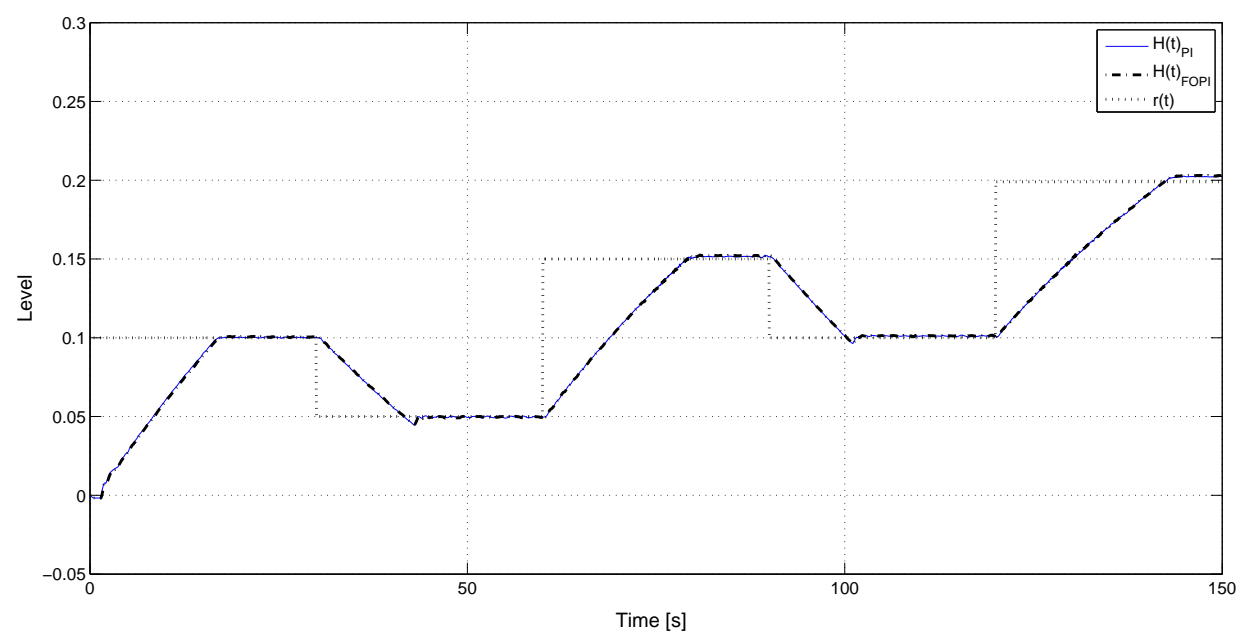

Fig. 10. Several step responses 
The corresponding error diagrams are shown in Figure 11. The most interesting diagram is presented in Figure 12, where the integrated absolute error is. There is the most visible difference between the integrated absolute error of response with the PI and FOPI controller. Unfortunately, the result of the FOPI controlling is a little worse than the PI controlling result.

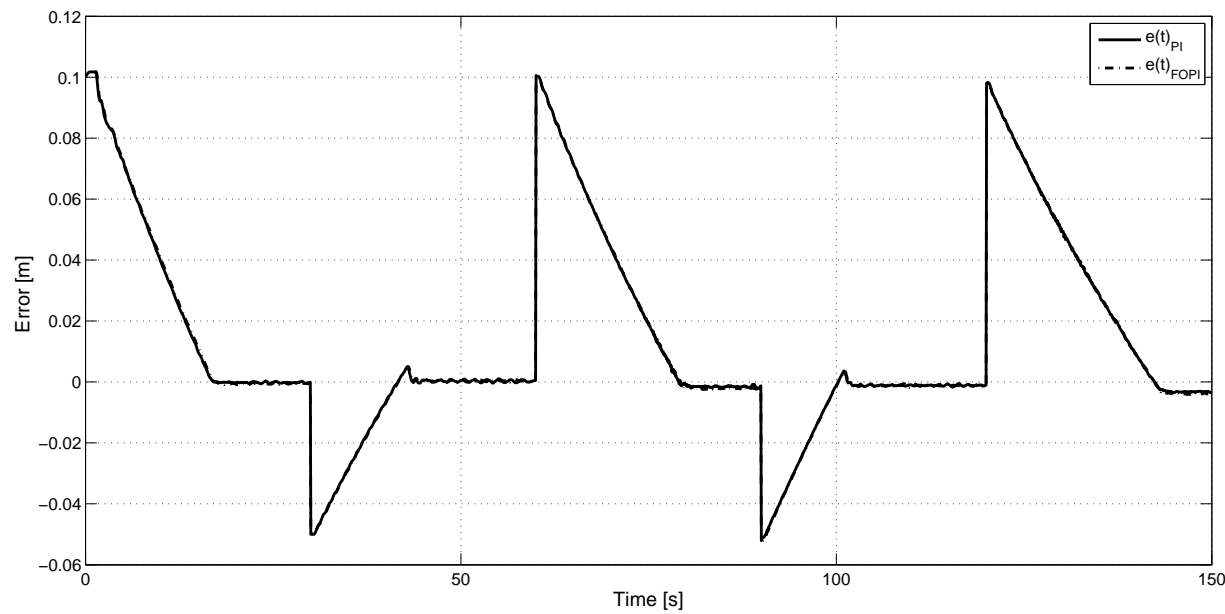

Fig. 11. The error for several step response

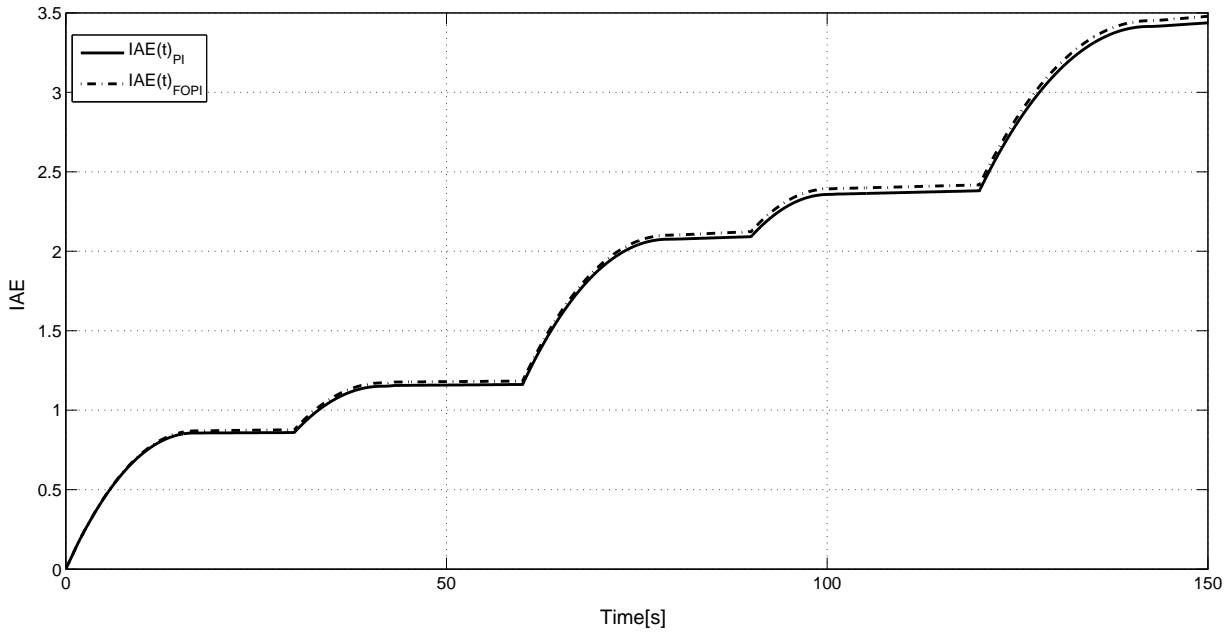

Fig. 12. The Integrated Absolute Error for several step responses 


\section{Conclusion}

The comparison of two types of PI controllers has been conducted (classical PI controller and non-integer order $\mathrm{PI}^{\lambda}$ controller, FOPI). The ways of developing the two controllers have been presented and implemented in a hydro-mechanical system. Results show that the noninteger order $\mathrm{PI}^{\lambda}$ controller gives almost as good effects as the traditional PI controller in such systems but in the same time the non-integer order controller is computationally more complex than the PI controller, therefore using non-integer order $\mathrm{PI}^{\lambda}$ controller to control even such simple integrating systems proves useless.

\section{Acknowledgements}

The work was realised in the scope of project titled „Design and application of noninteger order subsystems in control systems". The project was financed by the National Science Centre on the base of decision no. DEC-2013/09/D/ST7/03960.

\section{References}

[1] Dziwiński T., Bauer W., Baranowski J., Piątek P., Zagórowska M., Robust Non-integer Order Controller for Air Heating Process Trainer, [in:] Latawiec K.J., Łukaniszyn M., Stanisławski R. (Eds.), Advances in Modelling and Control of Non-integer-Order Systems, 2015, 249-256.

[2] Bauer W., Dziwiński T., Baranowski J., Piątek P., Zagórowska M., Comparison of Performance Indices for Tuning of $\mathrm{PI}^{\lambda} \mathrm{D}^{\mu}$ Controller for Magnetic Levitation System, [in:] Latawiec K.J., Łukaniszyn M., Stanisławski R. (Eds.), Advances in Modelling and Control of Non-integer-Order Systems, 2015, 125-133.

[3] Dziwiński T., Bauer W., Baranowski J., Piątek P., Zagórowska M., Robust non-integer order controller for air heater. $19^{\text {th }}$ International Conference on Methods and Models in Automation and Robotics, MMAR, 2015.

[4] Bauer W., Baranowski J., Mitowski W., Non-integer order PI $\alpha$ D $\mu$ control ICU-MM. Theory \& Appl. of Non-integer Order Syst. Lecture Notes in Electrical Engineering, 257:295-303, Springer, Heidelberg, 2013

[5] Rosół M., Application of nonlinear control methods for tanks system (in Polish). Pomiary, Automatyka, Kontrola, 7/8:30-34, 2001.

[6] Oustaloup A., La commande CRONE: commande robuste d'ordre non entier Hermes, 1991.

[7] Oustaloup A., F. Levron F., Mathieu B., Nanot F.M., Frequency-band complex noninteger differentiator: characterization and synthesis. Circuits and Systems I: Fundamental Theory and Applications, IEEE Transactions on, 47(1):25-39, 2000. 